\title{
Dynamic Analysis of Redundant Parallel Manipulator
}

\author{
Li Qing ${ }^{1, a}$,Ren YongXin ${ }^{2, b}$, Zhang Jing ${ }^{2, c}$ \\ ${ }^{1}$ China Academy of Machinery Science and Technology, NO.2 Shouti South Road,Haidian District, \\ Beijing, 100044,China \\ ${ }^{2}$ Advanced Manufacture Technology Center of China Academy of Machinery Science Technology, \\ No.18 Xue Qing Road, Haidian District, Beijing, 100083,China, \\ aliqing313152@126.com, ${ }^{b}$ renyongxin75@126.com, 'cmailzhjing@126.com
}

Keywords: Redundant Drive; Parallel Mechanism; Freedom; Dynamic Analysis

Abstract: The structure of 5-UPS/PRPU redundant parallel manipulator is introduced. The degree of freedom and redundant type of this manipulator is analyzed. And, the dynamic equations of five UPS branches and a PRPU branch are established with Lagrange Method. This study provides theoretical basis for the control system of 5-UPS / PRPU redundant parallel manipulator.

\section{Introduction}

There are some problems of parallel mechanism, such as, little work space, singular configuration, and some others ${ }^{[1,2]}$. Redundant drive, introduced to the parallel mechanism, can solve these problems, effectively ${ }^{[3]}$. Redundant drive means that the degree of freedom of parallel mechanism is less than the number of drives. So the control system of redundant parallel mechanism is much complex than the one of non-redundant parallel mechanism. Currently, redundant parallel mechanism has become a hot research topic at home and abroad ${ }^{[4]}$.

The study of redundant parallel mechanism includes two aspects, that are, redundant parallel mechanism dynamic analysis and redundant parallel mechanism control system. The former aspect is to solve redundant parallel mechanism driving force and its optimization [5], mainly. The latter one is to solve the implementation of control system and its real-time problem [6]. In this paper, 5-UPS/PRPU redundant parallel manipulator is introduced. And, the dynamic equations of this manipulator are established with Lagrange Method, to provide theoretical basis of driving force configuration and optimization.

\section{Structure Analysis of 5-UPS/PRPU Redundant Parallel Manipulator}

5-UPS/PRPU parallel manipulator includes five branches of UPS. The five branches of UPS link the top platform and the moving platform, as figure 1 shown. What's more, the middle branch is PRPU, as constraint. Pose adjustment of the moving platform is achieved by driving the five branches, through changing the length of them, to make this manipulator work. 


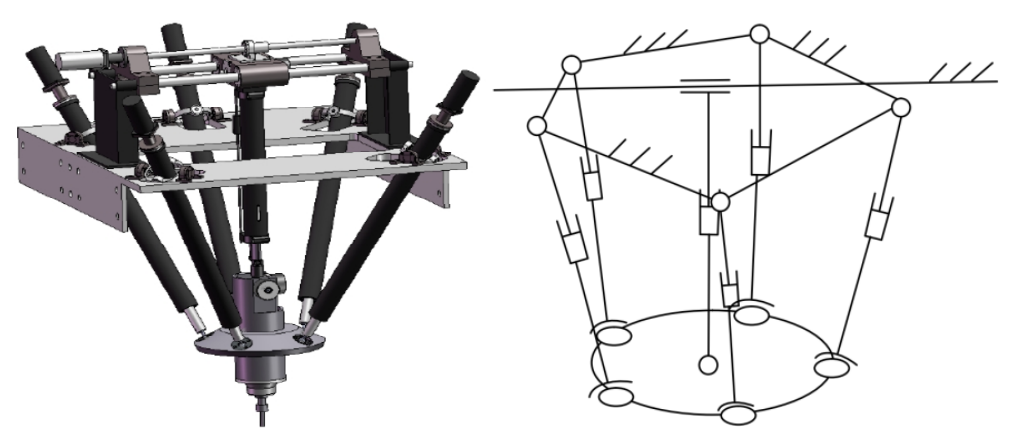

Figure 1 5-UPS/PRPU Parallel Manipulator

The PRPU branch of 5-UPS/PRPU parallel manipulator has two functions. First, to limit the moving platform's degree of freedom, that is, to limit its rotation around itself. Second, to fix measurement sensors to obtain the moving platform position posture data, providing real-time data to the closed loop control system. While, the PRPU branch is as an passive restraint branch. And, there are some problem, such as, large frictional force, large inertia. These problems lead the 5-UPS/PRPU parallel manipulator to appear creeping phenomenon, to reduce the work accuracy. To eliminate this phenomenon, one driver is added on the PRPU branch, making it be a 5-UPS/PRPU redundant parallel manipulator.

\section{DOF Analysis of 5-UPS / PRPU Redundant Parallel Manipulator}

As is shown in formula (1), it is to calculate the degree of freedom of 5-UPS / PRPU redundant parallel manipulator.

$$
\mathrm{M}=6(\mathrm{n}-\mathrm{g}-1)+\sum_{\mathrm{i}=1}^{\mathrm{g}} \mathrm{f}_{\mathrm{i}}
$$

Where, M is the degree of freedom of 5-UPS / PRPU redundant parallel mani-

Pulator.

$\mathrm{n}$ is the total number of components.

$\mathrm{g}$ is the total number of joints.

fi is the degree of freedom of ith joint.

The 5-UPS/PRPU redundant parallel manipulator's total number of components is $n=15$. The total number of joints is $g=19$. And, the total number of degree of freedom is $\sum_{\mathrm{i}-1}^{\mathrm{g}} \mathrm{f}_{\mathrm{i}}=35$. So, the degree of freedom can be obtained as followed formula.

$$
\mathrm{M}=6(\mathrm{n}-\mathrm{g}-1)+\sum_{\mathrm{i}=1}^{\mathrm{g}} \mathrm{f}_{\mathrm{i}}=6 \times(15-19-1)+35=5
$$

With calculation, the5-UPS/PRPU redundant parallel manipulator's degrees of freedom of is 5.

\section{Dynamic Analysis of 5-UPS/PRPU Redundant Parallel Manipulator}

The common methods to study dynamic analysis of parallel manipulator are Lagrange Method, Kane Method, Gauss Method, Newton - Euler Method and the Principle of Virtual Work Method. Because of the Lagrange Method with the good point of concision in modeling and solving parallel mechanism. The Lagrange Method is used in this paper. With generalized coordinates, dynamic analysis of 5-UPS / PRPU parallel mechanism is studied. The number of dynamic analysis equations is equal to the number of degree of freedom of the mechanism. And dynamic analysis 
equations is without analyzing the internal force, while dynamic equations are built with conservation of energy.

The dynamic model of 5-UPS / PRPU parallel mechanism is established as formula (3) shown.

$$
\frac{\mathrm{d}}{\mathrm{dt}}\left(\frac{\partial \mathrm{L}}{\partial \mathrm{q}_{\mathrm{k}}}\right)-\frac{\partial \mathrm{L}}{\mathrm{q}_{\mathrm{k}}}=\mathrm{Q}_{\mathrm{k}}(\mathrm{k}=1,2, \ldots, \mathrm{N})
$$

Where, $\mathrm{L}=\mathrm{T}-\mathrm{V}$ is the Lagrange Equation.

$\mathrm{q}_{\mathrm{k}}=\left[{ }^{\mathrm{A}} \mathrm{X}_{\mathrm{o}},{ }^{\mathrm{A}} \mathrm{X}_{\mathrm{o}},{ }^{\mathrm{A}} \mathrm{X}_{\mathrm{o}}, \alpha, \beta\right]^{\mathrm{T}}$ is the generalized coordinate.

$\dot{\mathrm{q}}_{\mathrm{k}}=\left[{ }^{\mathrm{A}} \dot{\mathrm{X}}_{\mathrm{o}},{ }^{\mathrm{A}} \dot{\mathrm{X}}_{\mathrm{o}},{ }^{\mathrm{A}} \dot{\mathrm{X}}_{\mathrm{o}}, \dot{\alpha}, \dot{\beta}\right]^{\mathrm{T}}$ is The first derivative of the generalized coordinate.

$Q_{k}$ is the non-conservative force in generalized coordinate $q_{k}$.

The kinetic energy of the 5-UPS / PRPU parallel mechanism is as formula (4) shown.

$$
\mathrm{T}=\sum_{\mathrm{i}-1}^{3} \mathrm{E}_{1}
$$

Where, E1 is the kinetic energy of the moving platform.

$\mathrm{E}_{2}$ is the kinetic energy of the UPS branch.

$\mathrm{E}_{3}$ is the kinetic energy of the PRPU branch.

The potential energy of the 5-UPS / PRPU parallel mechanism is as the formula (5) shown.

$$
\mathrm{V}=\sum_{\mathrm{i}=1}^{3} \mathrm{~V}_{1}
$$

Where, V1 is the potential energy of the moving platform.

$\mathrm{V}_{2}$ is the potential energy of the UPS branch.

$\mathrm{V}_{3}$ is the potential energy of the PRPU branch.

With formula (4) and (5), the kinetic - potential energy function is as formula (6) shown.

$$
\mathrm{L}=\mathrm{T}-\mathrm{V}=\sum_{\mathrm{i}=1}^{3}\left(\mathrm{E}_{1}-\mathrm{V}_{1}\right)
$$

Taking formula (6) into formula (3), the left equation expression can be obtained as follows.

$$
\mathrm{Q}_{\text {left }}=\left[\mathrm{Q}_{\text {left }_{\mathrm{x}}}, \mathrm{Q}_{\text {lefty }}, \mathrm{Q}_{\text {leftz }}, \mathrm{Q}_{\text {left } \alpha}, \mathrm{Q}_{\text {left } \beta}\right]^{\mathrm{T}}
$$

Since the drive joints of 5-UPS/PRPU parallel mechanism are moving pair, the driving force can be expressed as formula (8) shown.

$$
\mathrm{f}=\left[\mathrm{f}_{1}, \mathrm{f}_{2}, \mathrm{f}_{3}, \mathrm{f}_{4}, \mathrm{f}_{5}, \mathrm{f}_{6}\right]^{\mathrm{T}}
$$

The external force of 5-UPS / PRPU parallel mechanism is transformed to the origin coordinate system. The formula (9) is obtained as follow.

$$
[\mathrm{F}, \mathrm{M}]^{\mathrm{T}}=\left[{ }^{\mathrm{A}} \mathrm{F}_{\mathrm{x}},{ }^{\mathrm{A}} \mathrm{F}_{\mathrm{y}},{ }^{\mathrm{A}} \mathrm{F}_{\mathrm{z}},{ }^{\mathrm{A}} \mathrm{M}_{\mathrm{x}},{ }^{\mathrm{A}} \mathrm{M}_{\mathrm{y}},{ }^{\mathrm{A}} \mathrm{M}_{\mathrm{z}}\right]
$$

5-UPS / PRPU parallel mechanism is driven by redundant driving force. The driving force of five UPS branch and one PRPU branch is as formula (10) shown.

$$
\mathrm{Q}_{1}=\mathrm{G}_{\mathrm{F} 6} \mathrm{f}
$$

The role of the external force can be expressed as formula (11) shown. 


$$
\mathrm{Q}_{2}=\mathrm{G}_{\mathrm{W}}[\mathrm{F}, \mathrm{M}]^{\mathrm{T}}
$$

With formula (10) and (11), the right expression of Lagrange dynamic equation is as follow.

$$
\mathrm{Q}_{\text {right }}=\mathrm{Q}_{1}+\mathrm{Q}_{2}=\mathrm{G}_{\mathrm{F} 6} \mathrm{f}+\mathrm{G}_{\mathrm{W}}[\mathrm{F}, \mathrm{M}]^{\mathrm{T}}
$$

Since $\mathrm{Q}_{\text {right }}=\mathrm{Q}_{\text {left }}$ the driving force of 5-UPS/PRPU redundant parallel mechanism is as formula (13) shown.

$$
\mathrm{G}_{\mathrm{F} 6} \mathrm{f}=\mathrm{Q}
$$

Where, $Q=Q_{\text {left }}-G_{W}[F, M]^{T}$ 。

Because $\mathrm{G}_{\mathrm{F} 6}$ 's generalized inverse matrix $\mathrm{G}_{\mathrm{F} 6}{ }^{-1}$ does not exist, 5-UPS/PRPU parallel mechanism can be driven by redundant driving force.

\section{Conclusion}

The redundant type and structure of 5-UPS/PRPU parallel manipulator are introduced in this paper. And, degree of freedom is analyzed to study the redundant driving. With Lagrange Method, dynamic equations of five UPS branches and a PRPU branch are established. This study provides theoretical basis for 5-UPS / PRPU redundant parallel manipulator control system .

\section{Acknowledgements}

This work was financially supported by Fund Project of International Science and technology cooperation projects (2013DFA71730).

\section{References}

[1] R. Neugebauer, M. Schwaar, S. Ihlenfeldt. New Approaches to Machine Structures to Overcome the Limits of Classical Parallel Structures [J]. CIRP Annals Manufacturing Technology, 2002, 51(1): 293-296.

[2] K. H. Hunt. Structural Kinematics of In-Parallel Actuated Robot Arm [J]. Mechanisms, Transmissions, and Automation in Design, 1983, 105: 705-712.

[3] O. Piccin, B. Bayle, B. Maurin, et al. Kinematic Modeling of a 5-DOF Parallel Mechanism for Semi-spherical Workspace [J]. Mechanism and Machine Theory, 2009, 44(8): 1485-1496.

[4] D. Stewar. A Platform with Six Degrees of Freedom [J]. I Mech E, 1965, 180(15): 371-386.

[5] S. Modic. Virtual Axis Machining the Shape of Things to Come [J]. Tools \& Production, 1994, 7: 13-14.

[6] A. Muller, T. Hufnagel. Model-based Control of Redundantly actuated Parallel Manipulators in Redundant Coordinates [J]. Robtics and Autonomous Systems, 2012, 60: 563-571. 